\title{
Protein kinases influence bovine oocyte competence during short-term treatment with recombinant human follicle stimulating hormone
}

\author{
Atef Ali and Marc-André Sirard \\ Centre de Recherche en Biologie de la Reproduction (CRBR), Department of Animal Science, Laval University, \\ Ste-Foy, Quebec, Canada, G1K 7P4
}

Correspondence should be addressed to M-A Sirard; Email: marc-andre.sirard@crbr.ulaval.ca

\begin{abstract}
The aim of this study was to investigate the effect of short-term treatment (first 2 or $\mathbf{6 h}$ ) with recombinant human folliclestimulating hormone ( $\mathrm{r}$-hFSH) during in vitro maturation (IVM) on the developmental competence of bovine oocytes. The roles of protein kinase $A$ (PKA) and protein kinase $C$ (PKC) (possibly involved in FSH response), were investigated using activators (Sp-cAMPS, PMA) or inhibitors (Rp-cAMPS, sphingosine) of these two protein kinases, respectively. The developmental competence of bovine oocytes was measured by the rate of blastocyst formation after in vitro fertilization (IVF). Our results showed that when cumulus-oocyte complexes (COCs) were cultured with r-hFSH for the first $6 \mathrm{~h}$, a highly significant $(P<0.0001)$ improvement is seen in blastocyst development rate as a proportion of oocytes in culture compared with those matured with r-hFSH for the first 2 or $24 \mathrm{~h}$. A transient exposure $(6 \mathrm{~h})$ to the highest dose $(100 \mu \mathrm{M})$ of forskolin (an activator of adenylate cyclase) increased $(P<0.05)$ the rate of blastocyst formation. But the PKA inhibitors (Rp-cAMPS) did not affect the stimulatory effects of $r$-hFSH on the blastocyst yield. However, stimulation of PKC by low doses of PMA (0.1-0.5 $\mu M)$ during short-term treatment, enhanced $(P<0.0001)$ the developmental capacity of oocytes, while sphingosine $($ a specific inhibitor of $\mathrm{PKC}$ ) inhibited $(P<\mathbf{0 . 0 5})$ the stimulatory effects of $r$-hFSH on the rate of blastocyst formation. Our results indicate that although the developmental capacity of bovine oocytes in vitro can be modulated by both the PKA, and the PKC pathways, the activation of PKC during short-term treatment can mimic the effect of r-hFSH on the cytoplasmic maturation in bovine oocytes in vitro.

Reproduction (2005) $130303-310$
\end{abstract}

\section{Introduction}

In mammals, primary oocytes spontaneously resume meiosis when liberated from antral ovarian follicles (Pincus \& Enzmann 1935, Edwards 1965). Resumption of meiosis leads to germinal vesicle breakdown (GVBD), the first meiotic reduction division, and arrest at metaphase II. The culmination of this process may be observed by the presence of the first polar body. Oocytes undergo a series of cytoplasmic changes leading to variable competence of the resulting embryo (Blondin et al. 2002). In addition, the synthesis and storage of certain forms of mRNA and protein during IVM and early embryonic development are thought to be necessary for further development (Motlik \& Fulka 1986, Thibault et al. 1987).

Although it seems difficult to influence oocytes during their maturational period to modulate their response for subsequent development, the alteration of basic maturation conditions by specific regulators can significantly affect oocyte competence as reflected by the morula and blastocyst yield after in vitro fertilization (IVF; Rose-Hellekant \& Bavister 1992). It is well known that the ability of in vitromatured oocytes to develop to the blastocyst stage is indicative of oocyte competence although not necessarily indicative of live birth potential, and dependent on the stage of oocyte and follicle growth (First et al. 1988).

Meiotic resumption of mammalian oocytes is triggered by the preovulatory gonadotropin surge in vivo (Baker 1982). Since gonadotropin receptors are absent from oocytes, follicle-stimulating hormone (FSH) promotes GVBD via an indirect mechanism mediated by the cumulus cells rather than by a direct action on the oocyte (Eppig \& Downs 1987). There is clear evidence showing that addition of FSH to serum free maturation medium initially delays meiotic nuclear maturation due to the increase in cyclic adenosine monophosphate (cAMP) in cumulus cells, while it enhances the developmental ability of the oocyte (Izadyar et al. 1998). The main signal 
transduction resulting from $\mathrm{FSH}$ binding to cumulusgranulosa cells is considered to be cAMP-protein kinase A (PKA) (Eppig \& Downs 1984). However, the results of some studies showed that protein kinase $\mathrm{C}$ (PKC) may also play an important role in the signal transduction of $\mathrm{FSH}$ (Aberdam \& Dekel 1985, Su et al. 1999).

Studies of PKA or PKC on further development of mammalian oocytes especially, in gonadotropin-induced processes are rather insufficient and the results contradictory due to the variation in oocytes. Some reports indicate that activation of PKC can induce GVBD in rat cumulus cell enclosed oocytes (CEOs) (Aberdam \& Dekel 1985) and rabbit (Yoshimura et al. 1992). But in mouse CEOs (Bornslager et al. 1986, Kimura 1996, Su et al. 1999) and cow denuded oocytes (DOs) (Homa 1991), PKC activators can inhibit polar body emission but only at high doses. At low doses, as in our study, the authors have seen a stimulation of maturation.

Recently, we have demonstrated that the effect of FSH on nuclear maturation and cumulus expansion is dependent on substrates present in IVM medium (Ali \& Sirard 2002a). Other work in our laboratory has shown that the presence of physiological concentrations of $17 \beta$-estradiol (E2) and r-hFSH during IVM of bovine oocytes in defined medium significantly improved oocyte quality as reflected by the increased proportion of oocytes developing to the blastocyst stages after IVF (Ali \& Sirard 2002b).

Recombinant gonadotropins have become commercially available; these are very pure sources of hormone that allow interpretation of the individual roles of FSH and $\mathrm{LH}$ without the hormone cross-contamination of pituitary, serum or urinary preparations. In this study, we evaluated the possible signaling pathways involved in the r-hFSH mediated oocyte maturation and subsequent embryonic development. The roles of PKA and PKC in further development of bovine oocytes after IVM in the presence or absence of $\mathrm{r}$-hFSH were investigated using activators or inhibitors of these two protein kinases for a defined period of time. The present experiments revealed the beneficial effect of both PKA and PKC during maturation on further development of bovine oocytes.

\section{Materials and Methods \\ Oocyte recovery and selection}

Cumulus-oocyte complexes (COCs) were obtained and prepared as described by Ali and Sirard (2002a, 2000b). Briefly, bovine ovaries at various stages of their estrous cycle were obtained from a slaughterhouse and transported to the laboratory. Cumulus-oocyte complexes (COCs) were recovered from $2-6 \mathrm{~mm}$ follicles. Only oocytes with at least four layers of compact cumulus cells and homogeneous cytoplasm were selected and then washed three times in modified synthetic oviduct fluid medium (m-SOF) (Tervit et al. 1972). The m-SOF medium used in this study was based upon the original formulation
(Tervit et al. 1972) with subsequent modifications (Gardner et al. 1994).

\section{In vitro maturation}

COCs were incubated in groups of ten in $50 \mu$ l droplets of maturation medium consisting of $\mathrm{m}$-SOF supplemented with $0.8 \%$ BSA-V (Sigma-Aldrich), MEM nonessential amino acids (Gibco, BRL, Burlington, Ontario, Canada), MEM essential amino acids (Gibco), $1.5 \mathrm{mM}$ glucose (Sigma-Aldrich), $1 \mathrm{mM}$ glutamine (Sigma-Aldrich) and $50 \mu \mathrm{g} / \mathrm{ml}$ gentamycin (Sigma-Aldrich). The droplets were covered with mineral oil (Sigma-Aldrich) and were preincubated under the maturation conditions for a minimum of $3 \mathrm{~h}\left(38.5^{\circ} \mathrm{C}, 5 \% \mathrm{CO}_{2}\right.$ in air with $100 \%$ humidity) and then incubated for $24 \mathrm{~h}$ after oocytes were added.

\section{In vitro fertilization}

The method used for in vitro fertilization was performed as described by Ali and Sirard (2002a, 2000b). Briefly, in vitro fertilization took place in droplets $(48 \mu \mathrm{l})$ composed of modified Tyrode lactate medium (Bavister et al. 1983), supplemented with $0.6 \%$ BSA fatty acid free (SigmaAldrich), $0.2 \mathrm{mM}$ pyruvic acid (Sigma-Aldrich), $2 \mu \mathrm{g} / \mathrm{ml}$ heparin (Sigma-Aldrich), and $50 \mu \mathrm{g} / \mathrm{ml}$ gentamycin containing five oocytes. All experiments were carried out using frozen semen from the same bull (Centre d'Insémination Artificielle du Québec; CIAQ, St-Hyacinthe, Québec, Canada). Spermatozoa were thawed at $35^{\circ} \mathrm{C}$ and washed in a discontinous Percoll gradient prepared by adding $2 \mathrm{ml}$ of $90 \%$ Percoll under $2 \mathrm{ml}$ of $45 \%$ Percoll in a 15-ml centrifuge tube (Falcon, Franklin Lakes, NJ, USA). The semen samples were added on top of the Percoll gradient and centrifuged at $700 \mathrm{~g}$ for $30 \mathrm{~min}$ at $26^{\circ} \mathrm{C}$. After removal of the supernatant, spermatozoa were resuspended in IVF medium, counted in a hemocytometer chamber and $2 \mu \mathrm{l}$ of sperm suspension (final concentration $=1 \times 10^{6}$ cells $/ \mathrm{ml}$ ) were added into each droplet. Incubation was carried out at $38.5^{\circ} \mathrm{C}$ in $5 \% \mathrm{CO}_{2}$ in air with saturated humidity for $15-18 \mathrm{~h}$.

\section{In vitro culture}

In all experiments, embryo culture took place in m-SOF (Gardner et al. 1994, 1997) under mineral oil in a humidified atmosphere of $5 \% \mathrm{CO} 2,7 \% \mathrm{O} 2$ at $38.5^{\circ} \mathrm{C}$. Between 15-18 h after insemination, presumptive zygotes were denuded of surrounding cumulus cells by repeated pipetting in phosphate buffered saline (PBS) and subsequently washed three times in PBS before being transferred to the culture droplets $(50 \mu \mathrm{l})$ in groups of 20-30 embryos. Cleavage was assessed after $72 \mathrm{~h}$ of culture and the number of embryos developing to the blastocyst stage was assessed on day 8. Blastocyst development is expressed as a proportion of oocytes in culture (blastocyst yield).

This study used a two-culture system as described previously (Ali \& Sirard 2002a, 2000b). The first system (SOFC1) medium contained $0.8 \%$ BSA, MEM nonessential 
amino acids, $1 \mathrm{mM}$ glutamine and $10 \mu \mathrm{M}$ EDTA (SigmaAldrich) for the first $72 \mathrm{~h}$. Then, the medium was replaced by the second system (SOFC2) containing $0.8 \%$ BSA, MEM nonessential amino acids, MEM essential amino acids and $1 \mathrm{mM}$ glutamine for the remaining $96 \mathrm{~h}$ of culture. To prevent toxic accumulation of ammonium as a result of amino acid degradation, SOFC2 medium was replaced after $72 \mathrm{~h}$ of culture.

\section{Experimental design}

All experiments were repeated three times.

\section{Experiment 1}

The objective of experiment 1 was to evaluate the effects of short-term treatment (first 2 or $6 \mathrm{~h}$ ) with r-hFSH (Gonal F; Serono, Ontario, Canada) during IVM on the developmental competence of bovine oocytes. COCs $(n=240)$ were cultured for the first 2 or $6 \mathrm{~h}$ in the presence of r-hFSH $(0.5 \mu \mathrm{g} / \mathrm{ml})$, washed carefully four times and then cultured for the last 18 or $22 \mathrm{~h}$ in. COCs cultured for $24 \mathrm{~h}$ in SOFBSA or SOFBSA $+\mathrm{r}-\mathrm{hFSH}$ served as the negative and positive control, respectively. Cumulus expansion was evaluated under a stereomicroscope at $18 \mathrm{~h}(-$, no expansion; + , intermediate or partial expansion; ++ , complete expansion) (Ali \& Sirard 2002a).

Experiment 2: effect of $C A M P+P K A$ pathway on the developmental competence of bovine oocytes

This experiment was designed to investigate whether the activation of cAMP + PKA was involved in the process of oocyte competence. COCs $(n=420)$ were cultured for the first $6 \mathrm{~h}$ in the maturation medium containing 5, 10, 50 and $100 \mu \mathrm{M}$ forskolin (Sigma-Aldrich), a stimulator of adenylate cyclase, and then the oocytes were transferred to a new medium without forskolin and cultured for a total of $24 \mathrm{~h}$. A solvent control treatment with ethanol $(0.1 \%)$ was included. Cumulus expansion was evaluated under a stereomicroscope at $18 \mathrm{~h}$ (Ali \& Sirard 2002a).

\section{Experiment 3}

This experiment was designed to determine whether the beneficial effect of r-hFSH on the developmental competence during a short-term treatment was mediated by protein kinase A (PKA). COCs $(n=420)$ were cultured in the presence $(1 \mathrm{mM})$ of the PKA inhibitors Rp-cAMPS (SigmaAldrich) or the PKA activators Sp-cAMPS (Sigma-Aldrich) (Aktas et al. 1995) for the first $6 \mathrm{~h}$ of culture with or without $\mathrm{r}-\mathrm{hFSH}$. After $6 \mathrm{~h}$ they were washed four times in SOF medium alone and twice in culture medium before they were cultured for the last $18 \mathrm{~h}$ in SOFBSA.

\section{Experiment 4}

These experiments were conducted to evaluate the effects of activation and/or inhibition of PKC on the developmental competence of bovine oocytes during a short-term treatment. (A) COCs $(n=600)$ were cultured with different concentrations $(0.05,0.1,0.5,1,5,10$, 50 and $100 \mu \mathrm{M}$ ) of phorbol myristate acetate (SigmaAldrich) (PMA, an activator of PKC) for the first $6 \mathrm{~h}$. (B) COCs $(n=360)$ were cultured with $r-h F S H$ for the first $6 \mathrm{~h}$ with or without $(1 \mu \mathrm{M})$ of sphingosine (SPH) (SigmaAldrich) (a specific inhibitor of PKC; Su et al. 1999). After $6 \mathrm{~h}$ COCs were washed four times in SOF alone and twice in culture medium before they were cultured for the last $18 \mathrm{~h}$ in SOFBSA. In addition, a group of oocytes were treated with $0.1 \%$ of ethanol to determine if the carrier used had an effect on the developmental competence of oocytes after IVF. Cumulus expansion was evaluated under a stereomicroscope at $18 \mathrm{~h}$ (Ali \& Sirard 2002a).

\section{Statistical analysis}

All experiments were repeated three times. Statistical analyses were carried out by ANOVA with Fisher's protected least significant difference (LSD) test using the STATVIEW program (SAS Institute, Inc., Cary, NC, USA). All percentage data were subjected to arcsine transformation before statistical analysis (Snedecor \& Cochran 1967). Data from three replicates were expressed as mean \pm S.E.M. A probability of $P<0.05$ was considered to be statistically significant.

\section{Results}

\section{Experiment 1}

After $72 \mathrm{~h}$ of culture, cleavage rates for oocytes cultured with r-hFSH for a short term (2 vs $6 \mathrm{~h}$ ) during IVM were similar and ranged from $70 \pm 2.6$ to $72 \pm 4.3$. However, 8 days after insemination, a higher $(P<0.0001)$ proportion of oocytes reached the blastocyst stage when COCs were cultured with r-hFSH for the first $6 \mathrm{~h}$ of IVM compared with other groups (Fig. 1).

In addition, the development of embryos to the blastocyst stage was improved by the addition of $\mathrm{r}$-hFSH for the first $2 \mathrm{~h}$ of IVM compared with the negative control (SOFBSA) $(P<0.05)$. COCs cultured with r-hFSH for a short-term period $(6 \mathrm{~h})$ showed signs of cumulus expansion only in the outer layer of the cumulus cells $(+)$ compared with those cultured for $24 \mathrm{~h}$ in the presence of $\mathrm{r}$-hFSH, which display a complete cumulus expansion $(++)$.

\section{Experiment 2}

When COCs were cultured with different concentrations of forskolin for the first $6 \mathrm{~h}$ of culture, the effect of supplementation of the maturation medium with forskolin depended on the forskolin concentration used. As shown in Fig. 2, COCs cultured with a lower concentration (5, 10 and $50 \mu \mathrm{M})$ of forskolin showed a lower 


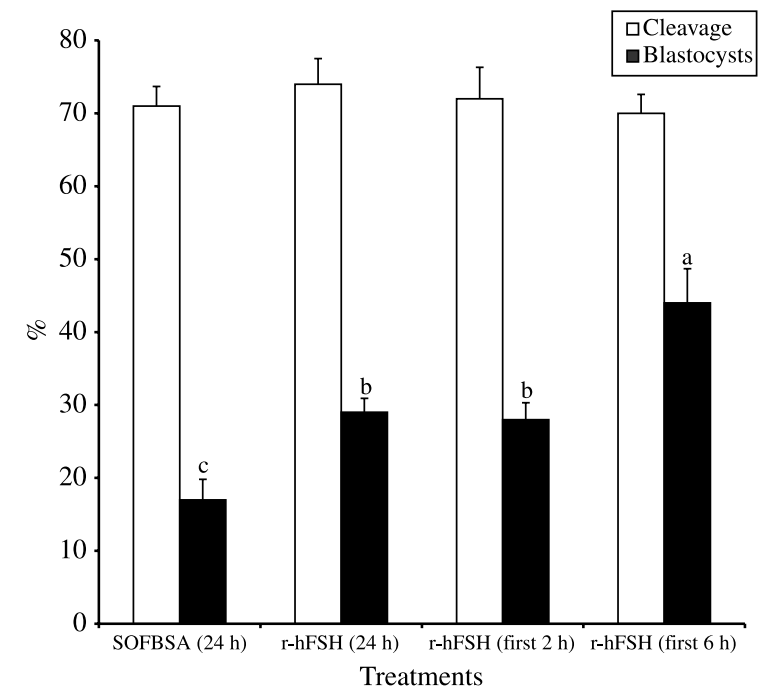

Figure 1 Effect of short-term treatment ( 2 or $6 \mathrm{~h}$ ) with recombinant human follicle-stimulating hormone (r-hFSH) during IVM on the developmental competence of bovine oocytes. Pooled data from three replicates (mean \pm S.E.M.). ${ }^{a-c}$ Values with different superscripts are significantly different $(P<0.05)$. SOFBSA, maturation medium without r-hFSH.

developmental rate compared with a higher concentration $(100 \mu \mathrm{M})$, which significantly $(P<0.05)$ enhanced the rate of blastocyst formation after IVF. The addition of $0.1 \%$ ethanol to the incubated COCs did not have a significant effect.

\section{Experiment 3}

COCs were cultured with the protein kinase A (PKA) inhibitors Rp-cAMPS or the PKA activators Sp-cAMPS for the first $6 \mathrm{~h}$ of culture with or without r-hFSH. After 8 days of insemination, the results show that Rp-cAMPS did not affect the stimulatory effects of r-hFSH on the blastocyst yield after IVF. In addition, no additive effect for Sp-cAMPS was found when added to the maturation medium in the presence of $\mathrm{r}$-hFSH during short-term treatment (Fig. 3).

\section{Experiment 4}

The PKC inhibitor, sphingosine suppressed the stimulatory effect of r-hFSH on the rate of blastocyst formation (Fig. 4). However, low doses $(0.1-0.5 \mu \mathrm{M})$ but not higher doses $(5-100 \mu \mathrm{M})$ of PMA enhanced $(P<0.0001)$ the rate of blastocyst formation (Fig. 5). In addition, COCs cultured with PMA at all concentrations used for a short-term treatment $(6 \mathrm{~h})$ showed signs of cumulus expansion in the outer layer of the cumulus cells compared with those cultured in maturation medium without PMA. The addition of $0.1 \%$ ethanol to the maturation medium did not

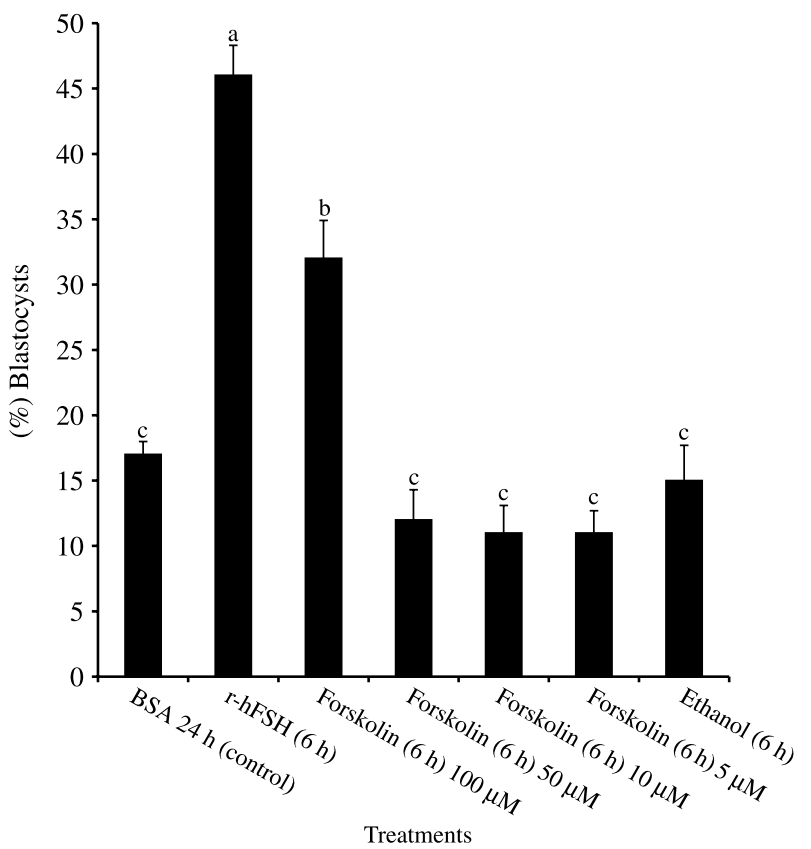

Figure 2 The effect of transient forskolin treatment on bovine oocyte developmental competence. Cumulus-oocyte complexes (COCs) were treated with various doses of forskolin for the first $6 \mathrm{~h}$, and then the COCs were transferred into a new medium without forskolin for another $18 \mathrm{~h}$. Pooled data from three replicates (mean \pm S.E.M.).

${ }^{a-c}$ Values with different superscripts are significantly different $(P<0.05)$. r-hFSH, recombinant human follicle-stimulating hormone.

have a significant effect on the rate of blastocyst formation (Fig. 5).

\section{Discussion}

The studies of the activation of the PKA or PKC in response to gonadotrophin are rather insufficient and the

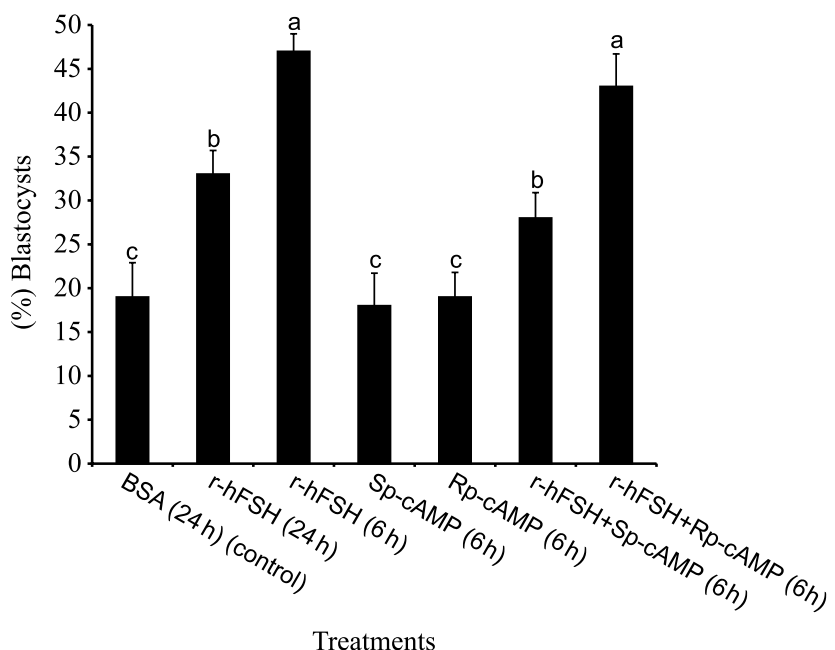

Figure 3 Effect of protein kinase A (PKA) inhibitors (Rp-cAMPS) or PKA stimulators (Sp-cAMPS) on recombinant human follicle-stimulating hormone ( $\mathrm{r}$-hFSH) during short-term treatment. Pooled data from three replicates (mean \pm S.E.M.). ${ }^{a-c}$ Values with different superscripts are significantly different $(P<0.05)$. 


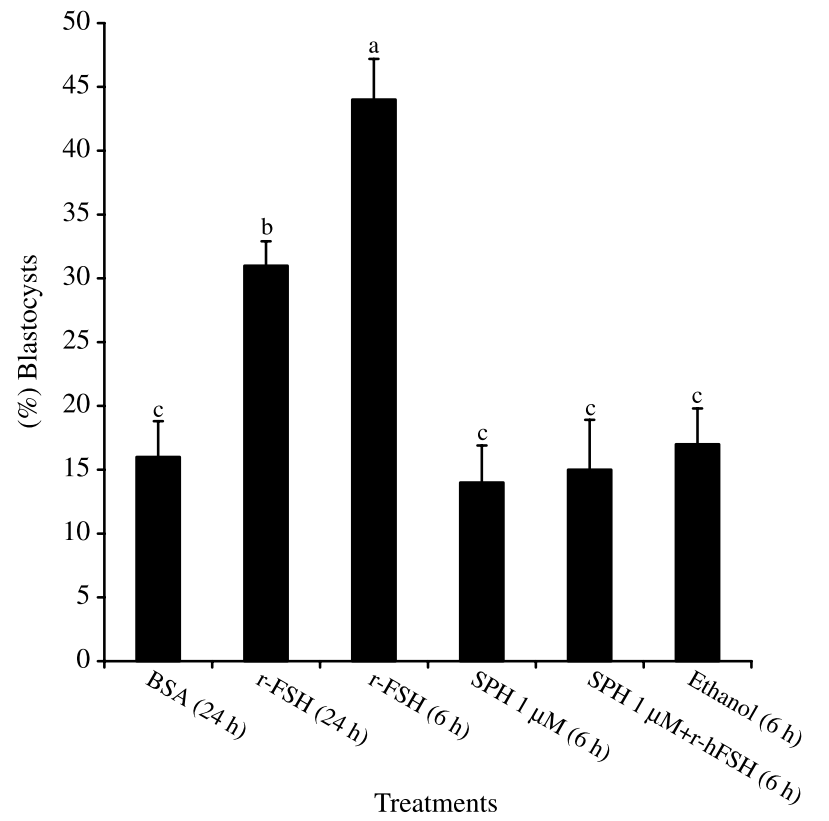

Figure 4 The effect of PKC inhibitor, sphingosine (SPH) on the developmental capacity of bovine oocyte. Cumulus-oocyte complexes (COCs) were cultured in the medium + recombinant human folliclestimulating hormone ( $r$-hFSH) with or without SPH. Pooled data from three replicates (mean \pm S.E.M.). ${ }^{a-c}$ Values with different superscripts are significantly different $(P<0.05)$.

results are often contradictory (Eppig \& Downs 1988, Colonna et al. 1997, Su et al. 1999). In order to discriminate the possible pathways involved in the gonadotropin mediated oocyte maturation, the roles of PKA and PKC in r-hFSH induced bovine oocyte competence were investigated. A recent study suggested that the mechanism of FSH-mediated mouse oocyte maturation is different from that of bovine or porcine (Lu et al. 2001). In the present study, it was surprising to see that Rp-cAMPS could not inhibit the beneficial effect of r-hFSH on oocyte competence and suggests that r-hFSH enhanced oocyte competence during short-term treatment was not strictly mediated by PKA.

The effects of CAMP and PKA on oocyte maturation are very complex. Stimulation of adenylate cyclase results in increased levels of cAMP in the oocyte. Forskolin, a direct stimulator of the catalytic subunit of adenylate cyclase, increases the synthesis of cAMP in both bovine COCs and denuded oocytes (Seamon et al. 1981, Bilodeau et al. 1993). In addition, forskolin treatment significantly increases the number of bovine oocytes in meiotic arrest (Homa 1988, Kuyt et al. 1988, Richard et al. 1997). The inhibitory effect of forskolin on oocyte maturation is increased when used in combination with 3-isobutyl-1methylxanthine (IBMX, 2 mM) (Bilodeau et al. 1993, Homa 1988). In the present study, there is an indication that COCs respond differently to relatively higher forskolin concentrations than lower concentrations. When data

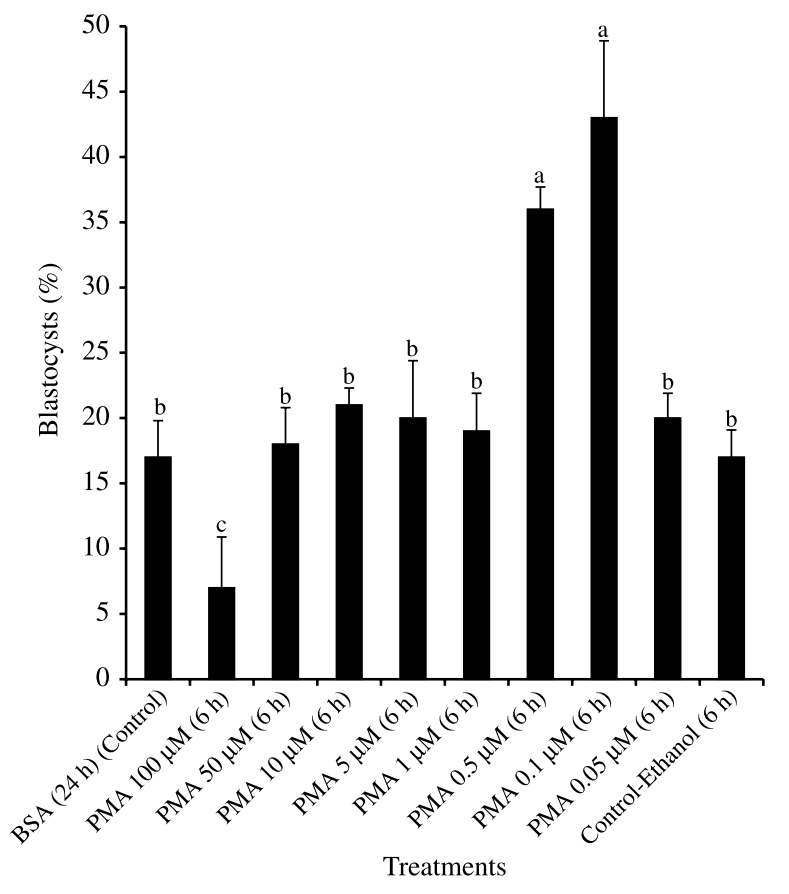

Figure 5 The effect of PKC activators (PMA) on bovine oocytes competence. Cumulus-oocyte complexes (COCs) were cultured in maturation medium with various doses of PMA during short-term treatment. Pooled data from three replicates (mean \pm S.E.M.).

${ }^{a-c}$ Values with different superscripts are significantly different $(P<0.05)$.

were analysed over all treatments, those treatments containing $100 \mu \mathrm{M}$ had an increased incidence of oocyte competence compared with that seen in treatments containing lower concentrations (Fig. 2). This paradox could be explained by the potential stimulatory effect of cAMP/PKA caused by higher forskolin level in cumulus and/or oocyte compartments that cause an increase in oocyte competence as reflected by the blastocyst stage after IVF. These results also suggest that cAMP has a physiological role in oocyte competence in bovine oocytes. Another important difference is the fact that treatment with $100 \mu \mathrm{M}$ forskolin for $6 \mathrm{~h}$ resulted in a cumulus expansion comparable to the $24 \mathrm{~h}$ treatment with $\mathrm{r}-\mathrm{hFSH}$ and therefore is different from the $6 \mathrm{~h}$ treatment with $\mathrm{r}$-hFSH where cumulus expansion is minimal.

It is believed that some effects of cAMP cannot be explained by activation of PKA (Kupperman et al. 1993, Dremier et al. 1997, Cass \& Meinkoth 1998, Cass et al. 1999). The data presented in our work imply that r-hFSH induced oocyte competence in bovine oocytes may be mediated by activation of the PKC pathway. PKC has been shown to be phosphorylated and activated by several cellular signalling pathways (Su et al. 1999). To determine whether r-hFSH might regulate the function of cumulus cells or oocytes via this pathway, COCs were cultured in the presence of $\mathrm{r}$-hFSH with or without sphingosine. Sphingosine is a potent and selective inhibitor of PKC 
(Homa 1991) and was used in this study to indirectly examine whether PKC could be activated by r-hFSH. Our present results showed that the inhibitory effect of sphingosine on r-hFSH as reflected by the rate of blastocyst formation, strongly supports the hypothesis that PKC is activated in the process of r-hFSH-mediated oocyte maturation. In order to further understand the relationship between PKC activation and oocyte competence, PMA, which can activate PKC in vitro, was used in these experiments. One of the surprising results of these experiments was the observation that PMA, at all concentrations, slightly stimulated cumulus expansion. Additionally, our results demonstrated that PMA at low levels enhanced oocyte competence as reflected by the blastocyst yield (Fig. 5). Moreover, PMA at a low concentration may mainly act on the cumulus cells, whereas at high concentration could also act on the oocytes (Su et al. 1999). We would like to suggest that PKC rather than PKA is involved in the short term r-hFSH induced oocyte competence. Moreover, the presence of low levels of PMA may sustain the right PKC activity in the COCs to improve cytoplasmic maturation as reflected by the blastocyst rates after IVF. We speculate that activation of PKC in the oocyte suppresses the developmental capacity of the oocyte, while stimulation within cumulus cells generates a positive trigger that leads to improved oocyte competence.

Our results also suggested that activation of PKC or sustained elevation of CAMP during short-term treatment could enhance oocyte competence. These results indicate that the follicular environment may maintain a favourable level of PKC activation in the bovine oocyte (as shown in this study) in order to improve cytoplasmic maturation. In our experiments, stimulators of PKC in COCs probably induced the cumulus to secrete positive factors to promote maturation of oocytes.

It was reported that $\mathrm{FSH}$ could stimulate the cumulus cells of the oocyte-cumulus complex to generate a meiosis-activating factor, e.g. $\mathrm{Ca}^{2+}$, a positive substance or heat-stable substance which positively overcomes the meiotic arrest with dibutyryl cyclic AMP or hypoxanthine and play a potent physiological role in triggering meiotic resumption by gonadotropin (He et al. 1997). It was proposed that this substance is meiosis-activating sterol (Byskov et al. 1995, 1997, Lu et al. 2001). The biochemical mechanisms mediating this process are still not very clear.

Based on the conditions employed in this study we hypothesize that increased developmental competence of bovine oocytes in response to r-hFSH occurs through the production of factor(s) that reach the oocyte through the cell-cell coupling pathways or that the coupling per se results in physiological changes in the oocyte. The beneficial effect of cumulus cells at the time of IVM on maturation and subsequent embryonic development is another interesting finding. Oocytes lack receptors for gonadotropin on their membrane. In fact, receptors for gonadotropins are expressed only in the somatic cells of the follicles (Eppig \& Downs 1987). Therefore, the hormones act on follicular cells and induced signals are transduced into the oocytes through gap junctions of extracellular mechanisms (Eppig \& Downs 1987).

Since FSH stimulates the production of IP3 (Su et al. 1999), it can be postulated that IP3 can induce calcium release from the IP3-sensitive calcium store in the cumulus cells. This calcium release may be related to the early transcriptional and translation events that are required for the stimulatory phase of gonadotrophin-mediated GVBD in cultured oocytes (Motlik et al. 1989, Farin \& Yang 1994). Furthermore, IP3 can also be transferred from the cumulus cells into the oocyte via gap junctions to mobilize the calcium within the oocyte (Homa 1995). In addition, calcium produced in cumulus cells can also be transferred into the oocytes through the same mechanism and induce the increase of calcium in the oocytes via a mechanism of calcium-induced calcium release (Berridge 1993).

This study is one of the first to report such high development rates of bovine oocytes after in vitro maturation using defined conditions, especially after short-term treatment with $\mathrm{r}$-hFSH. These results indicate a beneficial effect when oocytes are matured with r-hFSH only for the first $6 \mathrm{~h}$. Furthermore, these results confirmed our recent findings that the presence of cumulus cells during IVM of the bovine oocyte might be necessary to express the competence of oocytes, but cumulus expansion is not required to improve competence; at least there is not a linear relationship between cumulus expansion and cytoplasmic maturation (Ali \& Sirard 2002a, 2000b). Additionally, to our knowledge we are the first to demonstrate that: 1) PMA-treated oocytes can go through GV, and that 2) PKC activation enhances oocyte competence. Under the present experimental conditions, our data show that the PKC pathway is implicated in r-hFSH, which improved bovine oocyte competence, especially after short-term treatment. These results indicate that the PKA and PKC pathways can modulate the maturation of bovine oocytes in vitro. A better understanding of the mechanism by which r-hFSH stimulates the oocytes can have important implications in the light of clinical interest in use of $\mathrm{r}$-hFSH in assisted reproduction protocols.

\section{Acknowledgement}

This work was supported by the Natural Sciences and Engineering Research Council of Canada (NSERC).

\section{References}

Aberdam E \& Dekel N 1985 Activators of protein C stimulate meiotic maturation of rat oocytes. Biochemical and Biophysical Research Communications 132 520-574.

Aktas H, Wheeler MB, First NL \& Leibfried-Rutledge ML 1995 Maintenance of meiotic arrest by increasing [CAMP]i may have 
physiological relevance in bovine oocytes. Journal of Reproduction and Fertility 105 237-245.

Ali A \& Sirard MA 2002a Effect of the absence or presence of various protein supplements on further development of bovine oocytes during in vitro maturation. Biology of Reproduction 66 901-905.

Ali A \& Sirard MA 2002b The effects of 17 beta-estradiol and protein supplement on the response to purified and recombinant follicle stimulating hormone in bovine oocytes. Zygote 10 65-71.

Baker TG 1982 Oogenesis and ovulation. In Reproduction in mammals, 2nd edn, pp 17-45. Eds CR Austin \& RV Short. Cambridge: Cambridge University Press.

Bavister BD, Leibfried LM \& Lieberman G 1983 Development of preimplantation embryos of the golden hamster in a defined culture medium. Biology of Reproduction 28 235-247.

Berridge MJ 1993 Inositol trisphophate and calcium signaling. Nature 361 315-325.

Bilodeau S, Fortier MA \& Sirard MA 1993 The effect of adenylate cyclase stimulation on meiotic resumption and cyclic AMP content of zona-free and cumulus-enclosed bovine oocytes in vitro. Journal of Reproduction and Fertility 97 5-11.

Blondin P, Bousquet D, Twagiramungu $H$, Barnes $F$ \& Sirard MA 2002 Manipulation of follicular development to produce developmentally competent bovine oocytes. Biology of Reproduction $6638-43$.

Bornslager EA, Mattel P \& Schults RM 1986 Involvement of cAMPdependent protein kinase and protein phosphorylation in regulation of mouse oocyte maturation. Developmental Biology 114 453-462.

Byskov AG, Andersen CY, Nordholm L, Thogersen H, Xia G, Wassmann O, Andersen JV, Guddal E \& Roed T 1995 Chemical structure of sterols that activate oocyte meiosis. Nature 374 559-562.

Byskov AG, Yding Andersen C, Hossaini A \& Guoliang X 1997 Cumulus cells of oocyte-cumulus complexes secrete a meiosisactivating substance when stimulated with FSH. Molecular Reproduction and Development 46 296-305.

Cass LA \& Meinkoth JL 1998 Differential effects of cyclic adenosine 3', 5'-monophosphate on p70 ribosomal kinase. Endocrinology 139 1991-1998.

Cass LA, Summers SA, Prendergast GV, Backer JM, Birnbaum MJ \& Meinkoth JL 1999 Protein kinase A-dependent and -independent signaling pathways contribute to cyclic AMP-stimulated proliferation. Molecular and Cellular Biology $195882-5891$.

Colonna R, Tatone C, Francione A, Rosati F, Callaini G, Corda D \& Di Francesco L 1997 Protein kinase C is required for the disappearance of MPF upon artificial activation in mouse eggs. Molecular Reproduction and Development 48 292-299.

Dremier S, Pohl V, Poteet-Smith C, Roger PP, Corbin J, Doskeland SO, Dumont JE \& Maenhaut C 1997 Activation of cyclic AMPdependent kinase is required but may not be sufficient to mimic cyclic AMP-dependent DNA synthesis and thyroglobulin expression in dog thyroid cells. Molecular and Cellular Biology $\mathbf{1 7}$ 6717-6726.

Edwards RG 1965 Maturation in vitro of mouse, sheep, cow, pig, rhesus monkey and human ovarian oocytes. Nature 208 349-351.

Eppig JJ \& Downs SM 1984 Chemical signals that regulate mammalian oocyte maturation. Biology of Reproduction 30 1-11.

Eppig JJ \& Downs SM 1987 The effect of hypoxanthine on mouse oocyte growth and development in vitro: maintenance of meiotic arrest and gonadotropin-induced oocyte maturation. Developmental Biology 119 313-321.

Eppig JJ \& Downs SM 1988 Gonadotrophin-induced murine oocyte maturation in vivo is not associated with decreased cyclic adenosine monophosphate in the oocyte-cumulus cell complex. Gamete Research 20 125-131.

Farin CE \& Yang L 1994 Inhibition of germinal vesicle breakdown in bovine oocytes by 5,6-dichloro-1-beta-D-ribofuranosylbenzimidazole (DRB). Molecular Reproduction and Development 37 284-292.
First NL, Leibfried-Rutledge ML \& Sirard MA 1988 Cytoplasmic control of oocyte maturation and species differences in the development of maturational competence. Progress in Clinical and Biological Research 267 1-46.

Gardner DK, Lane M, Spitzer A \& Batt PA 1994 Enhanced rates of cleavage and development for sheep zygotes cultured to the blastocyst stage in vitro in the absence of serum and somatic cells: amino acids, vitamins, and culturing embryos in groups stimulate development. Biology of Reproduction 50 390-400.

Gardner DK, Lane MW \& Lane M 1997 Bovine blastocyst cell number is increased by culture with EDTA for the first $72 \mathrm{~h}$ of development form the zygote. Theriogenology 47278.

He CL, Damiani P, Parys JB \& Fissore RA 1997 Calcium, calcium release receptors, and meiotic resumption in bovine oocytes. Biology of Reproduction 57 1245-1255.

Homa ST 1988 Effects of cyclic AMP on the spontaneous meiotic maturation of cumulus-free bovine oocytes cultured in chemically defined medium. The Journal of Experimental Zoology $\mathbf{2 4 8}$ $222-231$.

Homa ST 1991 Neomycin, an inhibitor of phosphoinositide hydrolysis, inhibits the resumption of bovine oocyte spontaneous meiotic maturation. The Journal of Experimental Zoology $\mathbf{2 5 8}$ 95-103.

Homa ST 1995 Calcium and meiotic maturation of the mammalian oocyte. Molecular Reproduction and Development 40 122-134.

Izadyar F, Zeinstra E \& Bevers MM 1998 Follicle-stimulating hormone and growth hormone act differently on nuclear maturation while both enhance development competence of in vitro matured bovine oocytes. Molecular Reproduction and Development $\mathbf{5 1}$ $339-345$.

Kimura H 1996 Studies on protein tyrosine phosphorylation in mouse oocyte maturation. Nippon Sanka Fujinka Gakkai Zasshi 48 $1149-1156$.

Kupperman E, Wen W \& Meinkoth JL 1993 Inhibition of thyrotropinstimulated DNA synthesis by microinjection of inhibitors of cellular Ras and cyclic AMP-dependent protein kinase. Molecular and Cellular Biology 13 4477-4484.

Kuyt JR, Kruip TA \& de Jong-Brink M 1988 Cytochemical localization of adenylate cyclase in bovine cumulus-oocyte complexes. Experimental Cell Research 174 139-145.

Lu Z, Xia G \& Zhang J 2001 Protein kinase C, rather than protein kinase $\mathrm{A}$ is involved in follicle-stimulating hormone-mediated meiotic resumption of mouse cumulus cell-enclosed oocytes in hypoxanthine-supplemented medium. Molecular and Cellular Endocrinology 182 225-232.

Motlik J \& Fulk J 1986 Factors affecting meiotic competence in pig oocytes. Theriogenology 25 87-96.

Motlik J, Fulka J Jr, Prochazka R, Rimkevicova Z, Kubelka M \& Fulka J 1989 RNA and protein synthesis requirements for the resumption of meiosis in rabbit oocytes: the role of cumulus cells. Reproduction, Nutrition, Development 29 601-609.

Pincus G \& Enzmann EV 1935 The comparative behavior of mammalian eggs in vivo and in vitro. The Journal of Experimental Medicine 62 665-675.

Richard FJ, Fortier MA \& Sirard MA 1997 Role of the cyclic adenosine monophosphate- dependent protein kinase in the control of meiotic resumption in bovine oocytes cultured with thecal cell monolayers. Biology of Reproduction $\mathbf{5 6}$ 1363-1369.

Rose-Hellekant TA \& Bavister BD 1992 Effect of oocyte maturation medium in vitro development of in vitro fertilized bovine embryos. Molecular Reproduction and Development 31 72-77.

Seamon KB, Padgett W \& Daly JW 1981 Forskolin: unique diterpene activator of adenylate cyclase in membrane and intact cells. PNAS $783363-3367$.

Snedecor GW \& Cochran WG 1967 In Statistical Methods, 6th edn, pp 327-329: lowa State University Press.

Su YQ, Xia GL, Byskov AG, Fu GD \& Yang CR 1999 Protein kinase C and intracellular calcium are involved in follicle-stimulating 
hormone-mediated meiotic resumption of cumulus cell-enclosed porcine oocytes in hypoxanthine-supplemented medium. Molecular Reproduction and Development 53 51-58.

Tervit HR, Whittingham DG \& Rowson LE 1972 Successful culture in vitro of sheep and cattle ova. The Journal of Reproduction and Fertility $30493-497$.

Thibault C, Szollosi D \& Gerard M 1987 Mammalian oocyte maturation. Reproduction, Nutrition, Development 27 865-896.

Yoshimura Y, Nakamura Y, Ando M, Jinno M, Nanno T, Oda T, Koyama N \& Shiokawa S 1992 Protein kinase C mediates gonadotrpin-releasing hormone agonist-induced meiotic maturation of follicle-enclosed rabbit oocytes. Biology of Reproduction 47 $118-125$

Received 17 June 2004

First decision 22 July 2004

Revised manuscript received 9 September 2004

Accepted 26 May 2005 